\section{Cryogenic Focused Ion Beam Characterization of Lithium Metal Anodes}

\author{
Jungwoo Z. Lee, ${ }^{\dagger, \perp}$ Thomas A. Wynn, ${ }^{\ddagger}, \perp$ Marshall A. Schroeder, ${ }^{\S}$ Judith Alvarado, ${ }^{\ddagger}$, Xuefeng Wang, ${ }^{\dagger \oplus}$ \\ Kang $\mathrm{Xu}^{\S}$ and Y. Shirley Meng ${ }^{*, f,+, \|_{\odot}}$ \\ ${ }^{\dagger}$ Department of NanoEngineering, University of California, San Diego, La Jolla, California 92093, United States \\ ${ }^{\ddagger}$ Materials Science and Engineering, University of California, San Diego, La Jolla, California 92093, United States \\ ${ }^{\S}$ Electrochemistry Branch, Sensors and Electron Devices Directorate, U.S. Army Research Laboratory, Adelphi, Maryland 20783, \\ United States \\ "Sustainable Power and Energy Center (SPEC), University of California, San Diego, La Jolla, California 92093, United States
}

Supporting Information

\begin{abstract}
Lithium metal is viewed as the ultimate battery anode because of its high theoretical capacity and low electrode potential, but its implementation has been limited by low Coulombic efficiency and dendrite formation above a critical current density. Determining the fundamental properties dictating lithium metal plating-stripping behavior is challenging because characterization techniques are limited by the sensitivity of lithium metal to damage by external probes, which regularly results in altered morphology and chemistry. Motivated by recent application of cryogenic transmission electron microscopy (cryo-TEM) to characterize lithium metal at the atomic scale, we explore the cryogenic focused ion beam (cryo-FIB) method as a quantitative
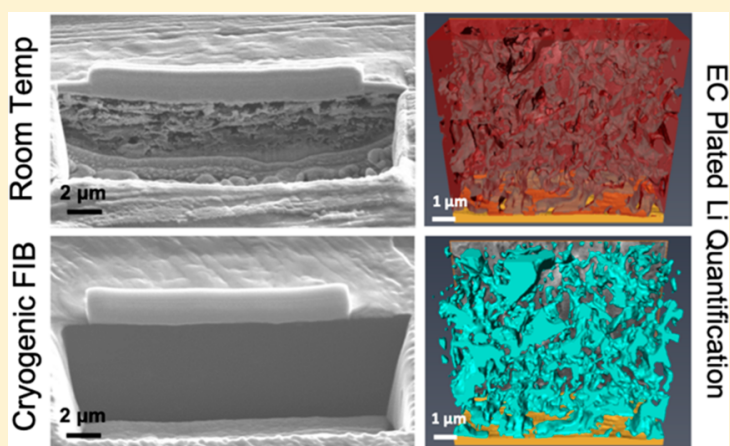
tool for characterizing the bulk morphology of electrochemically deposited lithium and as a technique that enables TEM observation of Li-metal/solid-state electrolyte interfaces. This work highlights the importance of cryo-FIB methodology for preparing sensitive battery materials and elucidates the impact of electrolyte selection on the density and morphology of plated lithium.
\end{abstract}

$\mathrm{T}$ here is significant motivation to enable lithium metal as an anode for rechargeable batteries because of its low electrode potential $(-3.04 \mathrm{~V}$ vs standard hydrogen electrode) and high theoretical specific capacity (3860 $\left.\mathrm{mAhg}^{-1}\right)$; however, despite nearly a half-century of research efforts, several significant challenges remain. Lithium's extreme reactivity with electrolytes prevents the formation of a stable solid electrolyte interphase (SEI), resulting in poor cycling efficiency. In addition, the electronic isolation of lithium during repeated cycling and formation of dendrites lead to reduced cycle life and major safety risks, respectively. While various techniques have been applied to mechanistically study lithiumion batteries, there are key limitations to characterizing lithium metal because of its intrinsic chemical reactivity, poor thermal stability, and low atomic number, making it prone to contamination and melting while exhibiting weak scattering characteristics for electrons and X-rays. ${ }^{1}$ Nonetheless, a technique that can elucidate the complex process surrounding lithium deposition and SEI formation with minimal sample perturbation has become an urgent necessity in order to develop mitigation strategies for enabling widespread adoption of lithium metal batteries.
Recently, cryogenic electron microscopy (cryo-EM) techniques, which have a rich history of use in visualizing the structure of biomolecules, ${ }^{2}$ have proven to be powerful tools for observing the fundamental structure and SEI composition of electrochemically deposited $\mathrm{Li}$ at the nanoscale. ${ }^{3,4}$ Specialized holders using liquid nitrogen cooling maintain the sample temperature at $-170{ }^{\circ} \mathrm{C}$ during imaging in order to minimize electron beam damage. This results in high imaging resolution and insight into the crystallography of $\mathrm{Li}$ dendrite growth and the effects of electrolyte chemistry on the SEI that are otherwise impossible to attain. However, this technique alone is limited to very thin specimens $(<100 \mathrm{~nm})$ deposited onto transmission electron microscopy (TEM) grids, preventing analysis of traditionally prepared and cycled bulk materials. In this work, we explore the ability of cryogenic focused ion beam (cryo-FIB) methods to process bulk lithium metal structures for advanced characterization.

Received: December 6, 2018

Accepted: January 10, 2019 
FIBs are versatile instruments for milling, imaging, and deposition used primarily for transmission electron microscopy (TEM) specimen preparation. These capabilities are accompanied by concerns of surface damage, redeposition, and preferential sputtering at high current density, as ion milling requires considerable ion beam-sample elastic collisions to transfer kinetic energy and eject material from the sample. ${ }^{5}$ Because of its low melting temperature, density, thermal conductivity, and shear modulus, lithium metal is especially sensitive to deleterious cascade effects and Ga-ion implantation. ${ }^{6}$ Baseline FIB milling performed on commercial Li foil samples at room temperature resulted in significant chemical and morphological damage even at reduced currents (Figure S1). Meanwhile, when the ion beam power was reduced to minimize such damage, there was not sufficient kinetic energy transfer to induce sputtering at reasonable rates. The mechanisms and properties influencing these phenomena are further discussed in the Supporting Information.

Cryo-FIB, primarily developed to prepare biological samples for cryo-EM, ${ }^{7}$ aims to minimize thermal damage and redeposition in order to preserve sensitive materials while maintaining high speed milling and instrument functionality. Recently, cryo-FIB techniques have been extending to lithium ion battery materials, employed to characterize lithium dendrites within a frozen liquid electrolyte. ${ }^{8,9}$ Schematic S1 outlines the key components of a cryo-FIB system where the sample is in thermal contact with a staged cooled with a chilled nitrogen gas source. Once the system achieves a stable cryogenic temperature, the cooled state can be maintained for several hours. Further experimental details are included in the Supporting Information. Using standard room-temperature milling processes, the commercial $\mathrm{Li}$ foil exhibits deleterious morphological modification due to local melting and quenching along with a high degree of $\mathrm{Ga}$ and $\mathrm{O}$ incorporation. Cleaning this surface at cryogenic temperatures helps minimize the $\mathrm{Ga}$ and $\mathrm{O}$ contamination, but morphological distortions still penetrate several hundreds of nanometers into the sample, serving as traps for redeposition of contaminated material. Only when the entire milling process is performed using a cryo-FIB at $-170{ }^{\circ} \mathrm{C}$ are the deleterious side effects minimized to preserve a dense, uniform, pristine $\mathrm{Li}$ foil (Figures 1 and S4).

FIB cross sections are often employed in battery material studies to correlate performance with morphology and density, but care must be taken to decouple electrochemical phenomena and artifacts from processing. At room temperature and high vacuum $\left(\sim 10^{-6} \mathrm{mbar}\right)$, the bulk lithium metal is more likely sensitive to local evaporation, leaving behind a network of SEI components and various redeposited compounds of higher melting points. Electrochemically plated $\mathrm{Li}$ in a standard carbonate electrolyte and milled using traditional room-temperature processes appears similar to previous representations of mossy $\mathrm{Li}$, while application of a cryo-FIB method shows that the more pristine state of dendritic features could be preserved (Figure S5). The cryogenic method also avoids mechanical deformation associated with "cutting" methods (i.e., microtome, scissors, etc.), thereby obtaining an accurate representation of the bulk film.

With the goal of enabling Li metal anodes for rechargeable batteries, there has been significant effort to develop and engineer new electrolytes to improve plating-deplating efficiencies to $>99.9 \%$ by controlling chemical and morpho-
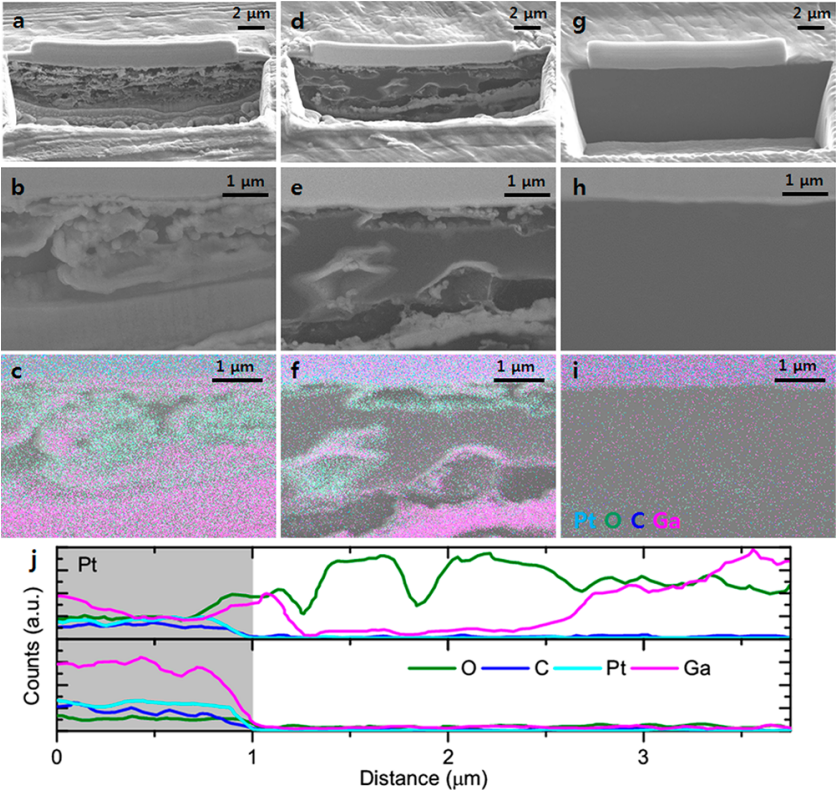

Figure 1. Scanning electron microscopy (SEM) images and energy dispersive spectroscopy (EDS) elemental mapping of cross sections of commercial $\mathrm{Li}$ metal foil $(\mathrm{a}-\mathrm{c})$ cross-sectioned and cleaned at room temperature, $(\mathrm{d}-\mathrm{f})$ cross-sectioned at room temperature and cleaned at cryogenic temperature, and $(g-i)$ cross-sectioned and cleaned at cryogenic temperature. ( $j$ ) Quantitative elemental line scans through room-temperature (top) and cryogenic temperature (bottom) cross sections.

logical inhomogeneities. Next-generation lithium metal batteries will require electrolytes with electrochemical stability windows beyond limits of ether- or carbonate-based systems to accommodate high-voltage cathode materials. ${ }^{10}$ Inspired by recent progress in high-concentration $(>3 \mathrm{M}$ ) electrolytes and the synergistic effect of using multiple salts, lithium bis(fluorosulfonyl)imide (LiFSI) and lithium bis(trifluoromethane)sulfonimide (LiTFSI) have been recently used in dimethoxyethane (DME) to improve Li-plating efficiency while expanding the electrolyte stability window to enable high nickel content cathode $\mathrm{LiNi}_{0.6} \mathrm{Mn}_{0.2} \mathrm{Co}_{0.2} \mathrm{O}_{2}$ (NMC-622) (unpublished results). The plating efficiency of this bisalt ether electrolyte ("BSEE", 4.6M LiFSI + 2.3M LiTFSI in DME) was compared with a concentrated single-salt ether electrolyte ("SSEE", 4.6M LiFSI-DME) and a carbonate baseline ("Gen II", 1.0M LiPF 6 EC/EMC 3:7) in $\mathrm{Li}$ vs $\mathrm{Cu}$ coin cells cycled at $0.5 \mathrm{~mA} / \mathrm{cm}^{2}$ to an areal capacity of $0.5 \mathrm{mAh} / \mathrm{cm}^{2}$ (Figure $2 \mathrm{a}$ ). The ether-based electrolytes exhibited significantly higher Li plating efficiencies compared to the carbonate-based electrolyte.

Because of the extreme chemical/electrochemical reactivity of lithium metal, the morphology and packing density of plated lithium are key factors that dictate cell efficiency and lifetime, as they define the true surface area of the reactive $\mathrm{Li}$ / electrolyte interface. From SEM images of cryo-FIB cross sections of the plated films, we can qualitatively describe the bulk plating behavior (Figure $2 \mathrm{~b}-\mathrm{d}$ ). Li plated in Gen II electrolyte consists of a thick and continuous, highly porous network with lithium metal branching and significant void spaces at the $\mathrm{Li} / \mathrm{Cu}$ foil interface propagating throughout the film. In contrast, the Li plated in SSEE and BSEE has reduced porosity and no observable dendritic Li. While this kind of qualitative analysis is powerful for elucidating trends in 

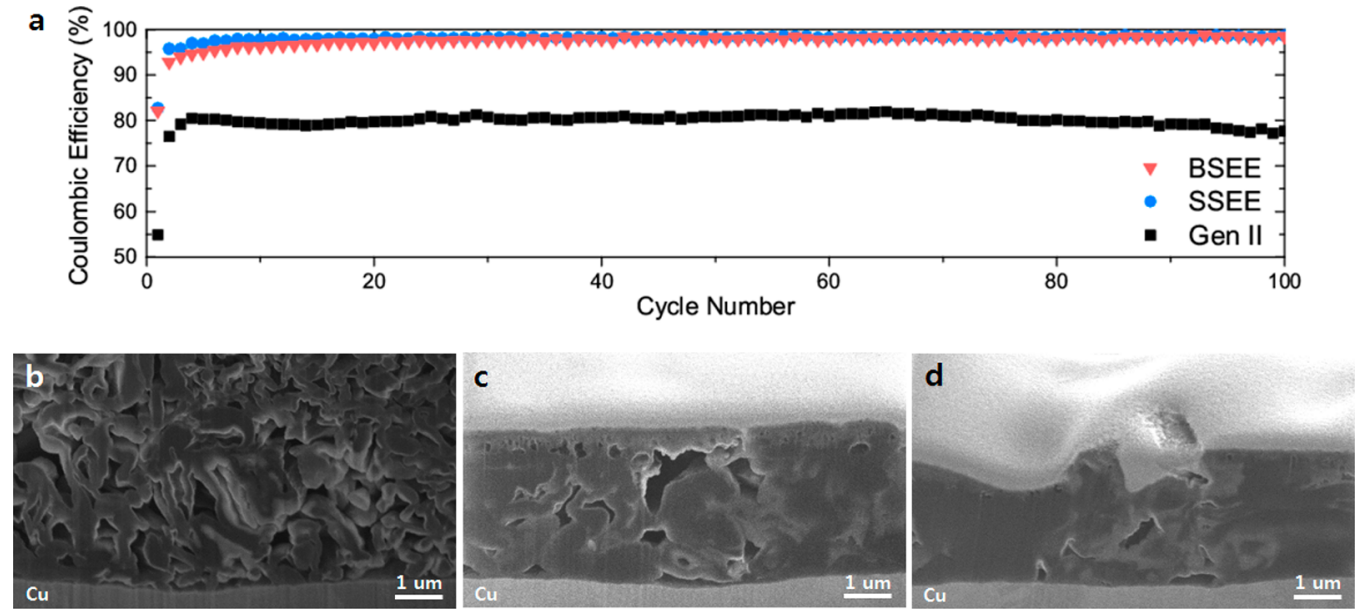

Figure 2. (a) Cycling performance of LillCu cells and cryo-FIB cross-sectional SEM images of the first cycle Li plate morphology for (b) 1.0 $\mathrm{M} \mathrm{LiPF}_{6} \mathrm{EC}_{\mathrm{E}} \mathrm{EMC}$ (Gen II), (c) 4.6M LiFSI-DME (SSEE), and (d) 4.6M LiFSI + 2.3M LiTFSI in DME (BSEE). Surfaces are coated with a protective FIB-deposited Pt layer.

morphology, packing density, and electrochemical performance, these are still highly localized observations. With this limitation in mind, we collected a series of high-resolution SEM cross-sectional images as we sequentially milled through a large area in the plane of the current collector (Schematic S2a). For each region of interest, gray scale intensity values were segmented to different regions, surfaces were generated, and quantitative analysis was performed using Amira-Avizo software (Schematic S2b).

Through this methodology, we create $3 \mathrm{D}$ reconstructions of electrochemically deposited $\mathrm{Li}$ that quantify a large total volume of sample with statistical microstructure information (Figure 3). We measure not only the total packing density of $\mathrm{Li}$ but also the size, shape, and distribution of voids. In
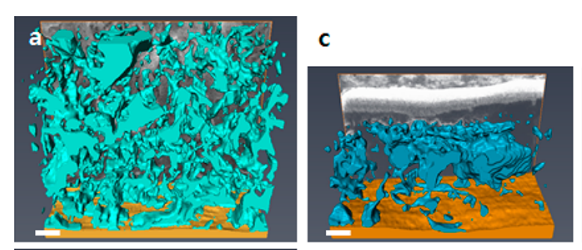

e

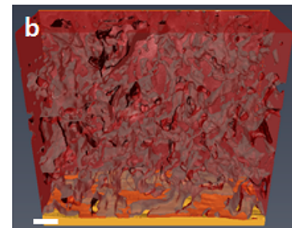

d

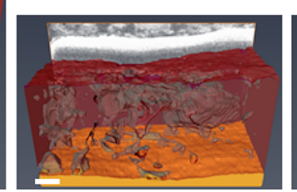

f
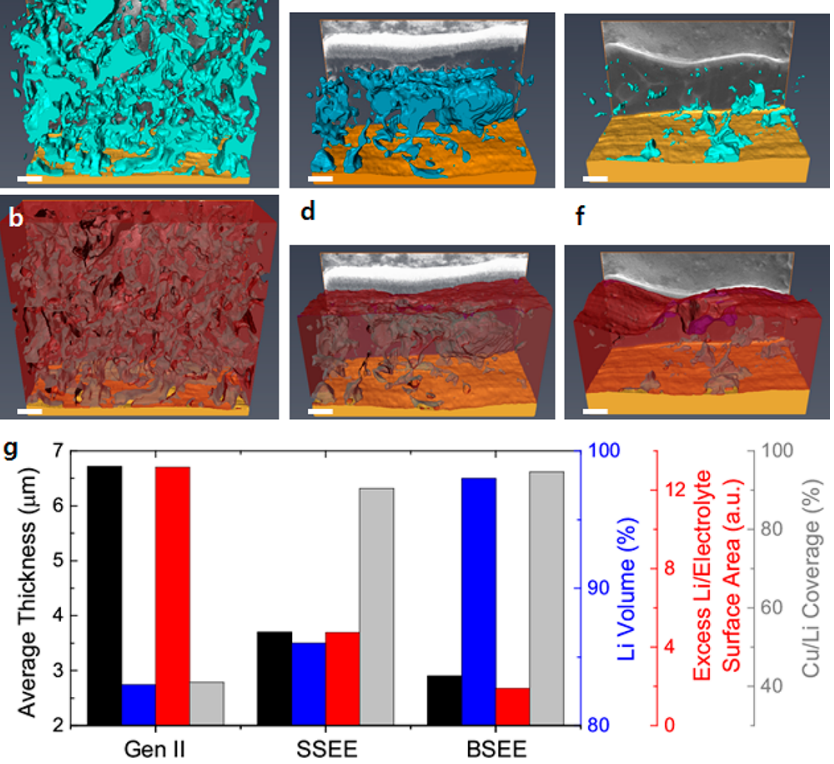

Figure 3. 3D reconstruction of voids (blue) and bulk $\mathrm{Li}$ metal (burgundy) with $1 \mu \mathrm{m}$ scale bar of first cycle electrochemically deposited Li in (a and b) 1.0 M LiPF6 EC: EMC (Gen II), (c and d) 4.6M LiFSI-DME (SSEE), and (e and f) $4.6 \mathrm{M} \mathrm{LiFSI}+2.3 \mathrm{M}$ LiTFSI in DME (BSEE) along with (g) statistical analysis. agreement with the trends extracted from the single crosssectional images, Li deposited in Gen II electrolyte exhibits dendritic growth and a large network of evenly distributed and connected pores. Conversely, Li deposited in SSEE and BSEE is significantly denser. Further, we can quantify the total volume of various components and the surface area between each phase to extract more precise values to evaluate the quality of plated Li from each electrolyte. While each sample has the same total quantity of $\mathrm{Li}$ plated, the electrolyte chemistry significantly alters the plating kinetics. Li plated in Gen II electrolyte has an average thickness of $6.7 \mu \mathrm{m}$ and $83 \%$ packing density. Li plated in high-concentration SSEE has a reduced average thickness of $3.7 \mu \mathrm{m}$ but only a marginally better packing density of $86 \%$. While the deposited Li in SSEE is much smoother with no dendrites, there are still large voids throughout the film and at the $\mathrm{Cu} / \mathrm{Li}$ interface. On the other hand, Li plated in the BSEE presents the thinnest $(2.9 \mu \mathrm{m})$ and densest $(98 \%)$ film. Note that the thickness values above are local, averaged from the sampled volumes, and may deviate from global film thicknesses. Further deviation from theoretical plating volume may be attributed to the presence of SEI components in the plated material (unpublished results).

Most importantly, we can now quantify the true $\mathrm{Li} /$ electrolyte interface surface area by normalizing it over the sample current collector surface area. For a perfectly dense sample, this ratio would be 1 (i.e., no excess surface induced by morphological features), and it is proportional to the increase in SEI surface area. The Li plated in Gen II electrolyte has a normalized $\mathrm{Li}$ /electrolyte area of 13.18 , as compared with the Li plated in SSEE of 4.7 and BSEE of 1.9. Thus, the Li plated in Gen II has a normalized Li/electrolyte surface area $\sim 7$ times greater than the plated Li from BSEE, significantly increasing the extent of parasitic surface reactions contributing to capacity degradation. Variation in the $\mathrm{Li} /$ electrolyte surface area may further enable deviation from theoretical plating densities, due to the modified availability for SEI volume.

An equally important metric is the Li nucleation coverage on the $\mathrm{Cu}$ current collector, as this will directly influence the growth kinetics and the probability of dendrite formation. For an ideal layer-by-layer growth mechanism resulting from a homogeneous current density, this coverage would be $100 \%$. For the Li plated in Gen II electrolyte, which exhibits dendritic 
growth, only $41 \%$ of the $\mathrm{Cu}$ current collector is plated with $\mathrm{Li}$, which increases to $90 \%$ and $95 \%$ for SSEE and BSEE, respectively. This suggests a synergistic mechanism between LiFSI and LiTFSI during Li plating to promote better current collector surface nucleation, more conformal deposition, and reduced surface area available for electrolyte side reactions.

Cryo-FIB was then applied to samples after the first stripping to $1 \mathrm{~V}$, further demonstrating the problematic morphology of the Li plated from Gen II electrolyte. The previous trends hold true where the $\mathrm{Li}$ plated and stripped in Gen II electrolyte leaves behind a thick layer of $\sim 550 \mathrm{~nm}$ porous $\mathrm{Li}$ with large voids at the $\mathrm{Cu}$ interface, while the $\mathrm{Li}$ plated and stripped in the BSEE leaves behind a dense, thin layer of $\sim 100 \mathrm{~nm}$ conformal to the $\mathrm{Cu}$ current collector (Figure 4). The density
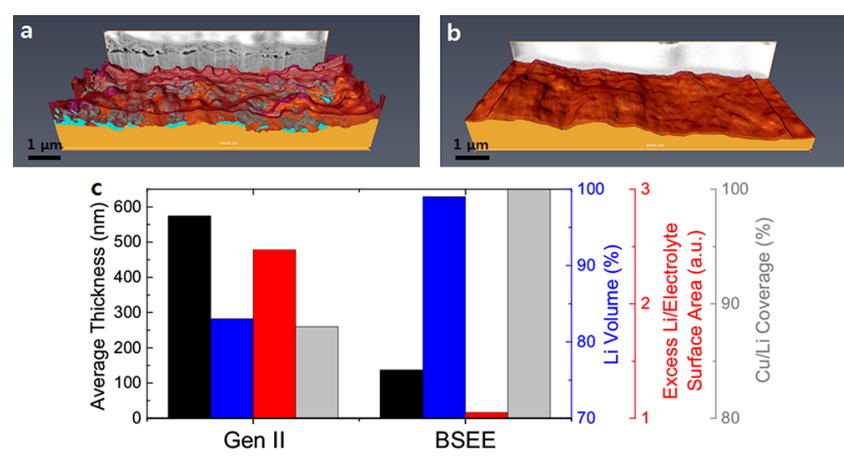

Figure 4. 3D morphology reconstruction of voids (blue) and bulk $\mathrm{Li}$ metal (burgundy) of first cycle electrochemically stripped $\mathrm{Li}$ in (a) 1.0 M LiPF6 EC: EMC (Gen II) and (b) 4.6M LiFSI + 2.3M LiTFSI in DME (BSEE) along with (c) statistical analysis.

and uniformity after stripping is important because this is now the surface structure for plating in subsequent cycles. The inhomogeneity of the Li plated and stripped in the Gen II electrolyte will result in uneven local current density, so these trends will be exacerbated over time, resulting in significant performance degradation.

Having demonstrated its ability to reliably analyze plated lithium metal, the cryo-FIB technique was extended to solidstate batteries and interfaces, which are currently considered a safer alternative to state-of-the-art nonaqueous electrolytebased batteries. Thin-film batteries consisting of a $\mathrm{LiCoO}_{2}$ (LCO) cathode, a lithium phosphorus oxynitride (LiPON) electrolyte, and a $\mathrm{Li}$ metal anode have shown excellent cyclability, ${ }^{11}$ though such solid-state interfaces-particularly the Li/solid-state electrolyte (SSE) interface-are poorly understood. We initially cross-sectioned the battery to clearly identify the $6.5 \mu \mathrm{m}$ LCO cathode, $2.0 \mu \mathrm{m}$ LiPON electrolyte, and 5-10 $\mu \mathrm{m}$ Li metal anode. Standard room-temperature FIB milling processes have been previously used to fabricate LCO and LiPON-based thin-film nanobatteries while minimizing damage and maintaining electrochemical activity. ${ }^{12,13}$ As previously discussed, the $\mathrm{Li}$ metal anode is prone to local morphological defects and $\mathrm{Ga}$ contamination with roomtemperature milling (Figure 5a). The cryo-FIB method minimizes these deleterious processing effects, revealing a pristine thin-film battery with dense layers along with smooth and conformal interfaces (Figure 5b). After a catastrophic event such as crushing or rupture causing the battery to short, we observe significant porosity at the Li/electrolyte interface and growth of the disordered LCO layer, both indicative of
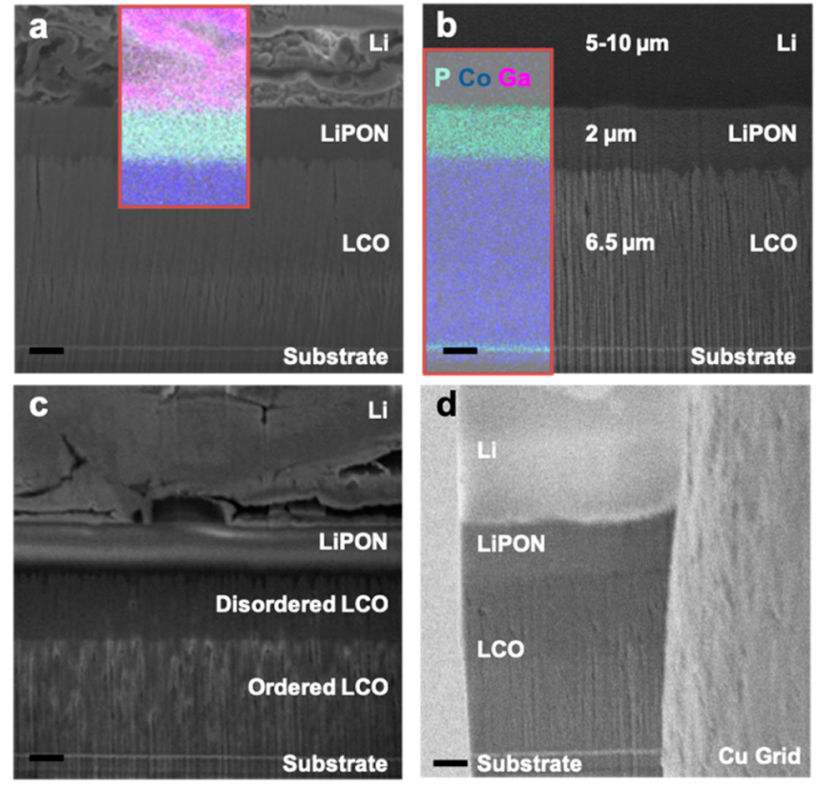

Figure 5. SEM image and EDS elemental map of cross section of pristine $\mathrm{Li}$ metal thin-film battery prepared at (a) room temperature and (b) under cryogenic conditions (layer thicknesses shown). (c) SEM image of sample after catastrophic failure. (d) TEM lamella of commercial $\mathrm{Li}$ metal thin-film battery to enable future studies of $\mathrm{Li}$ metal/electrolyte interfaces. Scale bars are 1 $\mu \mathrm{m}$.

increased interfacial resistance and poor cell performance (Figure 5c). ${ }^{12,14}$

With the goal of enabling cryo-TEM analysis of $\mathrm{Li}$ metal anodes and $\mathrm{Li}$ /SSE interfaces, we demonstrate cryo-FIB TEM lift-outs of Li metal and Li metal solid-state batteries (Figures S6 and 5d). Despite Li metal's sensitivity to local heating, control of beam conditions and sample temperature enables fabrication of TEM lamellae, which can then be thinned to electron transparency $(<100 \mathrm{~nm})$. The key challenge is to consider and balance the chemical, thermal, electrical, and mechanical properties of all the components, which will influence their interaction with the ion and electron beams. After achieving cryogenic temperatures, the TEM lift-out procedure may proceed as at room temperature with the caveat that it must be brought to room temperature to deposit Pt. ${ }^{6}$ Afterward, samples prepared with this process are brought back to room temperature, removed from the FIB under vacuum using an air-free quick loader (FEI), and stored in an Arpurged glovebox. Transfer into the TEM and cooling of the Li metal lamella were carried out using techniques described previously. ${ }^{3}$ Fast Fourier transform (FFT) analysis confirms that the Li foil maintains its polycrystalline structure during the cryo-FIB sample preparation process; however, some surface oxidation is observed (Figure S7). This is likely due to the room-temperature sample-transfer process, as even high vacuum and glovebox environments still contain trace amounts of oxygen, and the lamella has an increased surface area-tovolume ratio. Going forward, it is best to maintain the sample at cryogenic temperatures even during the sample-transfer processes to minimize these reactions. ${ }^{8}$

In this work, we demonstrate the importance and potential of cryogenic FIB methods for processing and characterizing sensitive battery materials. Under standard processing conditions at room temperature, lithium metal is prone to 
damage from $\mathrm{Ga}^{+}$implantation and local heating that distorts chemical and morphological observations. 3D reconstructions of electrochemically deposited and plated $\mathrm{Li}$ enable quantitative evaluation of electrolyte impact on the nucleation, density, and morphology of plated lithium, which directly dictate longterm cycling performance. Further, we extend the cryo-FIB technique to process and analyze TEM lamella of bulk lithium metal and lithium metal solid-state batteries, which when coupled with cryo-TEM, offer the potential to elucidate the complex structural and chemical phenomena at $\mathrm{Li}$ metal/ electrolyte interfaces.

\section{ASSOCIATED CONTENT}

\section{S Supporting Information}

The Supporting Information is available free of charge on the ACS Publications website at DOI: 10.1021/acsenergylett.8b02381.

Detailed description of the experimental methods and additional data and figures (PDF)

\section{AUTHOR INFORMATION}

\section{Corresponding Author}

*E-mail: shmeng@ucsd.edu, shirleymeng@ucsd.edu.

\section{ORCID}

Xuefeng Wang: 0000-0001-9666-8942

Y. Shirley Meng: 0000-0001-8936-8845

\section{Author Contributions}

${ }^{\perp}$ J.Z.L. and T.A.W. contributed equally.

\section{Notes}

The authors declare no competing financial interest.

\section{ACKNOWLEDGMENTS}

The authors gratefully acknowledge funding support from the U.S. Department of Energy, Office of Basic Energy Sciences, under Award Number DE-SC0002357 (program manager Dr. Jane Zhu). FIB was performed at the San Diego Nanotechnology Infrastructure (SDNI), a member of the National Nanotechnology Coordinated Infrastructure, which is supported by the National Science Foundation (Grant ECCS1542148). TEM was performed at the UC Irvine Materials Research Institute (IMRI). The authors thank D. GuyBouyssou and M. Proust from STMicroelectronics for supplying EnFilm batteries. M.S, J.A, K.X. are grateful for financial support from the Assistant Secretary for Energy Efficiency and Renewable Energy, Office of Vehicle Technologies of the U.S. Department of Energy under the Battery500 Consortium.

\section{REFERENCES}

(1) Lin, D.; Liu, Y.; Cui, Y. Reviving the Lithium Metal Anode for High-Energy Batteries. Nat. Nanotechnol. 2017, 12, 194-206.

(2) Murata, K.; Wolf, M. Cryo-Electron Microscopy for Structural Analysis of Dynamic Biological Macromolecules. Biochim. Biophys. Acta, Gen. Subj. 2018, 1862, 324-334.

(3) Wang, X.; Zhang, M.; Alvarado, J.; Wang, S.; Sina, M.; Lu, B.; Bouwer, J.; Xu, W.; Xiao, J.; Zhang, J. G.; et al. New Insights on the Structure of Electrochemically Deposited Lithium Metal and Its Solid Electrolyte Interphases via Cryogenic TEM. Nano Lett. 2017, 17, 7606-7612.

(4) Li, Y.; Li, Y.; Pei, A.; Yan, K.; Sun, Y.; Wu, C. L.; Joubert, L. M.; Chin, R.; Koh, A. L.; Yu, Y.; et al. Atomic Structure of Sensitive
Battery Materials and Interfaces Revealed by Cryo-Electron Microscopy. Science 2017, 358, 506-510.

(5) Rubanov, S.; Munroe, P. R. Investigation of the Structure of Damage Layers in TEM Samples Prepared Using a Focused Ion Beam. J. Mater. Sci. Lett. 2001, 20, 1181-1183.

(6) Giannuzzi, L. A.; Stevie, F. A. Introduction to Focused Ion Beams: Instrumentation, Theory, Techniques and Practice; Springer: New York, 2005.

(7) Rigort, A.; Plitzko, J. M. Cryo-Focused-Ion-Beam Applications in Structural Biology. Arch. Biochem. Biophys. 2015, 581, 122-130.

(8) Zachman, M. J.; Asenath-Smith, E.; Estroff, L. A.; Kourkoutis, L. F. Site-Specific Preparation of Intact Solid-Liquid Interfaces by LabelFree in Situ Localization and Cryo-Focused Ion Beam Lift-Out. Microsc. Microanal. 2016, 22, 1338-1349.

(9) Zachman, M. J.; Tu, Z.; Choudhury, S.; Archer, L. A.; Kourkoutis, L. F. Cryo-STEM Mapping of Solid-Liquid Interfaces and Dendrites in Lithium-Metal Batteries. Nature 2018, 560, 345349.

(10) Alvarado, J.; Schroeder, M. A.; Zhang, M.; Borodin, O.; Gobrogge, E.; Olguin, M.; Ding, M. S.; Gobet, M.; Greenbaum, S.; Meng, Y. S.; et al. A Carbonate-Free, Sulfone-Based Electrolyte for High-Voltage Li-Ion Batteries. Mater. Today 2018, 21, 341-353.

(11) Bates, J. B.; Dudney, N. J.; Neudecker, B.; Ueda, A.; Evans, C. D. Thin-Film Lithium and Lithium-Ion Batteries. Solid State Ionics 2000, 135, 33-45.

(12) Wang, Z.; Santhanagopalan, D.; Zhang, W.; Wang, F.; Xin, H. L.; He, K.; Li, J.; Dudney, N.; Meng, Y. S. In Situ STEM-EELS Observation of Nanoscale Interfacial Phenomena in All-Solid-State Batteries. Nano Lett. 2016, 16, 3760-3767.

(13) Lee, J. Z.; Wynn, T. A.; Meng, Y. S.; Santhanagopalan, D. Focused Ion Beam Fabrication of LiPON-Based Solid-State LithiumIon Nanobatteries for in Situ Testing. J. Visualized Exp. 2018, 133, No. e56259.

(14) Wang, Z.; Lee, J. Z.; Xin, H. L.; Han, L.; Grillon, N.; GuyBouyssou, D.; Bouyssou, E.; Proust, M.; Meng, Y. S. Effects of Cathode Electrolyte Interfacial (CEI) Layer on Long Term Cycling of All-Solid-State Thin-Film Batteries. J. Power Sources 2016, 324, 342348. 